\title{
LA EDUCACIÓN PARA EL DESARROLLO SOSTENIBLE EN LA UNIVERSIDAD BOLIVIANA. PERCEPCIONES DEL PROFESORADO
}

\author{
Education for Sustainable Development in the context \\ of higher education in Bolivia. Perceptions of university \\ professors
}

Lorena I. LITZNER ORDÓÑEZ, Werner RIEß

Pädagogische Hochschule Freiburg Kunzenweg. Alemania.

lorena.litzner@ph-freiburg.de; riess@ph-freiburg.de

bttps://orcid.org/0000-0003-3202-6830; https://orcid.org/0000-0002-3657-3359

Fecha de recepción: septiembre de 2018

Fecha de aceptación: enero de 2019

RESUMEN

El desarrollo sostenible es, hoy por hoy, uno de los principios rectores fundamentales dentro de la educación en general, y se constituye en tarea y desafío para la educación superior en particular. La educación superior para el desarrollo sostenible (ESDS) tiene la función de transmitir y desarrollar conocimientos, competencias, valores y actitudes que empoderen y motiven a los estudiantes a contribuir activamente con el desarrollo sostenible. El presente estudio empírico sobre el statu quo de la ESDS en Bolivia tiene como objetivo identificar las distintas variables del proceso de enseñanza-aprendizaje desde la perspectiva de los docentes universitarios; a saber, aspectos personales, aspectos curriculares, tanto de contenido como metodológicos, y aspectos estructurales e institucionales. Los resultados constituyen un esfuerzo en busca de operacionalizar la educación para el desarrollo sostenible (EDS) en la educación universitaria, con el objetivo ulterior de generar nuevas perspectivas para su conceptualización, implementación e institucionalización en el contexto universitario boliviano. 
LORENA I. LITZNER ORDÓÑEZ Y WERNER RIEß LA EDUCACIÓN PARA EL DESARROLLO SOSTENIBLE EN LA UNIVERSIDAD BOLIVIANA. PERCEPCIONES DEL PROFESORADO

Palabras clave: educación para el desarrollo sostenible; investigación empírica; educación superior; educación universitaria.

\section{ABSTRACT}

Sustainable development is, now days, one of most fundamental guiding principles in Education in general, and constitutes a task and challenge for higher education in particular. Higher Education for Sustainable Development (HESD) aims to transmit knowledge and develop competences, values and attitudes that empower and motivate students to actively contribute to sustainable development. The present empirical study was carried out on the status quo of education for sustainable development (EDS) in the context of Bolivian higher education. In the focus of the study were different structural elements of the teaching-learning process, analyzed from the perspective of the university professors. These were personal, curricular, methodological and finally structural and institutional aspects of the learning process. The results are an effort to operationalize the ESD in university education, with the ultimate goal of generating new perspectives for its conceptualization, implementation and institutionalization in the context of Bolivian higher education.

Key words: education for sustainable development; empirical research; higher education.

\section{INTRODUCCIÓN}

El concepto de desarrollo sostenible ${ }^{1}$ aplicado a la Educación tiene como principal objetivo brindar a todas las personas la oportunidad de adquirir los conocimientos, valores, y modelos de comportamiento, necesarios para adoptar estilos de vida en equidad y alcanzar transformaciones sociales positivas y duraderas. La Educación para el Desarrollo Sostenible (en adelante EDS) confronta a los estudiantes de forma constructiva con los problemas más serios que desafían a la humanidad; a saber, la explosión demográfica, las modificaciones en los ciclos de vida, la pérdida dramática de biodiversidad, la sobreexplotación de los recursos naturales y la problemática del agua dulce (Rieß, 2010) entre otros. Esta problemática, que es tan variada como compleja, requiere de cambios estructurales y acercamientos concretos, tarea que sin duda alguna es un reto para la educación en general y para la educación universitaria en particular. El desafío que plantea la EDS se centra en lograr incorporar en el proceso de enseñanza-aprendizaje, métodos participativos que motiven a los estudiantes a adquirir los conocimientos, las competencias, las actitudes y los valores necesarios para forjar un futuro sostenible. Es así, que el incorporar

1. Concepto acuñado en la Conferencia de las Naciones Unidas sobre el Medio Humano en Estocolmo en 1972, consolidado y oficializado a través de los principios 3, 4 y 8 de la Declaración de Río sobre el Medio Ambiente y el Desarrollo en 1992 (BID, 1993). 

PERCEPCIONES DEL PROFESORADO

los objetivos de una EDS efectiva, se convierte en una de las metas principales de la educación universitaria actual, con la finalidad de lograr una conciencia y un compromiso socio-ambiental, tanto en los estudiantes como en los demás actores del proceso educativo, para que éstos tengan la capacidad de identificar, evaluar y abordar los problemas y desafíos del desarrollo sostenible.

En el contexto de la educación superior o educación universitaria, el desafío es doble, ya que la universidad, como espacio de formación de los futuros actores sociales, tiene, por un lado, la responsabilidad de transmitir conocimientos, competencias y valores, y por el otro, la de facilitar e incentivar la generación de conocimientos nuevos e innovaciones científicas. El docente universitario se convierte en actor fundamental, y agente promotor y motivador de una conciencia ambiental en los estudiantes, quienes a su vez serán nuevos agentes de cambio encargados de romper las estructuras paradigmáticas incompatibles con la(s) solución(es) sustentable(s) del dilema ambiental.

\section{LA EDUCACIÓN PARA EL DESARROLlo SOSTENIBLE (EDS)}

Desarrollo sostenible es aquel "desarrollo que satisface las necesidades actuales, sin poner en riesgo, que las futuras generaciones puedan satisfacer sus propias necesidades» (Hauff, 1987, p. 46). El aspecto central del concepto de desarrollo sostenible enfatiza la así llamada multidimensionalidad (Rieß, 2010) de dicho precepto; vale decir, la convicción de que la protección de los recursos naturales, la responsabilidad social y la productividad económica son aspectos de una trilogía inseparable, interactuante e interdependiente. La EDS adapta para sí la multidimensionalidad de dicho concepto y piensa en la ecología, en la economía y en el ámbito social como un sistema, como una red o un network (De Haan y Harenberg, 1999).

El concepto de sustentabilidad se ha enraizado de tal forma en todos los ámbitos de conocimiento y comportamiento del ser humano, que las Ciencias de la Educación absorben dicho concepto, se apropian de él y le otorgan la calidad de principio fundamental, con carácter rector, transversal e inter y transdisciplinario. La Agenda 21, documento promulgado en la Conferencia de Río el año 1992, enlaza por primera vez el ámbito de la Educación con el concepto de Desarrollo Sostenible (Hauenschild y Rode, 2013). En su capítulo 36 Art. 3 establece:

La educación es de importancia crítica para promover el desarrollo sostenible y aumentar la capacidad de las poblaciones para abordar cuestiones ambientales y de desarrollo. La educación es igualmente fundamental para adquirir conciencia, valores y actitudes, técnicas y comportamientos ecológicos y éticos en consonancia con el desarrollo sostenible y que favorezcan la participación pública efectiva en el proceso de adopción de decisiones (ONU, 1992, p. 1).

La EDS enriquece la educación ambiental clásica (Lukman y Glavic, 2007), enfatizando la justicia y equidad inter e intrageneracional, subrayando la 
LORENA I. LITZNER ORDÓÑEZ Y WERNER RIEß

LA EDUCACIÓN PARA EL DESARROLLO SOSTENIBLE EN LA UNIVERSIDAD BOLIVIANA. PERCEPCIONES DEL PROFESORADO

multidimensionalidad de principio de sustentabilidad, respaldando la responsabilidad global, respetando la diversidad de culturas e incentivando la necesidad de participación política y social (Rieß, 2010). La EDS tiene como objetivo cualificar a las personas para identificar los problemas de un desarrollo no sostenible y para poder actuar y contrarrestar sus consecuencias negativas, con el fin de que las generaciones presentes y futuras tengan la oportunidad de alcanzar un «buen vivir ${ }^{2}$.

El concepto de EDS no es nuevo, más bien debe ser entendido como la continuación y complemento de los conceptos preexistentes de Educación Ambiental (en adelante EA) ${ }^{3}$, Pedagogía Ambiental (Grässel, 2009) y Ecopedagogía. En el espacio educativo latinoamericano, sobre todo a partir de la Conferencia de Tesalónica (1997), existe un debate y una tensión debido al «reemplazo o defunción de la EA, en favor de una EDS «(Mora Penagos, 2009, p. 18). En éste entendido, autores como Sauvé (2006), González Gaudiano (2006, 2008), Irwin (2008), entre otros, sostienen que el programa político y económico del desarrollo sostenible invade el campo de la educación y la torna en un «instrumento para un proyecto político-económico no discutido, sino indiscutible» (Sauvé, 2006, p. 89) operacionalizando a la EA como una herramienta para el desarrollo sostenible (Sauvé, 2006).

Uno de los impulsos internacionales más importantes en favor de la EDS fue sin duda la aprobación de la Década de la Educación para el Desarrollo Sostenible (DEDS) en el período del 2005 al 2014. Desde entonces, la EDS ha adquirido una legitimidad política, formal e integral, estableciendo que la sostenibilidad es tarea de todos y que debe introducirse y anclarse en todas las áreas y en todos los niveles de la educación. Concluido el período de la Década de la EDS, la Asamblea General de las Naciones Unidas adoptó, en septiembre de 2015, la Agenda Global post-2015 (2015-2030) para el Desarrollo Sostenible, presentando una lista de 17 Objetivos de Desarrollo Sostenible (ODS) que describen y aglomeran los principales desafíos de una vida sostenible, pacífica, próspera y justa para las generaciones actuales y venideras. La meta 4.7 de los ODS determina la necesidad de

garantizar que todos los alumnos adquieran los conocimientos teóricos y prácticos necesarios para promover el desarrollo sostenible, entre otras cosas mediante la educación para el desarrollo sostenible y la adopción de estilos de vida sostenibles, los derechos humanos, la igualdad de género, la promoción de una cultura de paz y no violencia, la ciudadanía mundial y la valoración de la diversidad cultural y de la contribución de la cultura al desarrollo sostenible, entre otros medios (UNESCO, 2017, p. 7)

2. Alusión al concepto del «buen vivir», del quechua "Sumak Kawsay» de los pueblos indígenas de Bolivia, Ecuador y Perú, que se refiere a la armonía de la humanidad con la "Pachamama» (Madre Tierra), y a la preservación de los sistemas y ciclos ecológicos de vida. Éste concepto acentúa la búsqueda de justicia, solidaridad y dignidad, el respeto por la diversidad, de una ética de la responsabilidad y una vida armoniosa en armonía con la naturaleza y las personas (Acosta, 2011, 2015; Boff, 2009).

3. La Educación Ambiental se gesta en la Cumbre del Medio Ambiente Humano celebrada en Estocolmo (1972) y en la Cumbre de Eduación Ambiental en Tbilisi (1978). 
En el contexto político, gubernamental e institucional, con los nuevos impulsos, iniciativas y esfuerzos a nivel internacional, existe un consenso generalizado en lo que respecta al rol de las universidades como centros de aprendizaje e investigación para el desarrollo sostenible; asignándoles la misión concreta de actuar como centros promotores y polos de actividad dentro de sus comunidades. La universidad no puede excusarse de cumplir con uno de los desafíos más grandes que afronta la humanidad, como es el del desarrollo sostenible (Leal Filho, 2010). Más aún, las instituciones de educación superior brindan el espacio real e ideal para la consolidación de la EDS; una EDS que va más allá de la mera difusión de conocimientos sobre temas del desarrollo sostenible (contenidos de aprendizaje) y que también promueve una nueva didáctica constructiva y enriquece los elementos estructurales del proceso de aprendizaje. En el contexto de la educación universitaria se habla de la Educación Superior para el Desarrollo Sostenible (ESDS).

\section{CONSIDERACIONES METODOLÓgICAS DE LA EDS}

Para lograr las metas que se plantea la EDS en la educación universitaria es necesario destacar el rol de los docentes como actores y agentes de cambio de la dinámica educativa. El desafío puntual del docente universitario, es lograr un cambio en la práctica docente, para que ésta se comprometa con la sostenibilidad. Parte de éste desafío consiste, debido a la característica transectorial y multidisciplinaria del dilema ambiental, en incorporar sus contenidos, objetivos y métodos en todas las disciplinas y áreas de conocimiento en el quehacer universitario. La EDS confronta a los estudiantes con problemas complejos, y les ayuda a emitir juicios valorativos y éticos, les insta a anticipar y analizar los efectos negativos posibles y a tomar decisiones de forma activa, participativa, democrática y sostenible. Las competencias de pensamiento sistémico, de anticipación, normativa, estratégica, de colaboración, de pensamiento crítico, de autoconciencia y la competencia integrada a la resolución de problemas (UNESCO, 2017) son competencias clave, cruciales para cumplir con los objetivos de la EDS (De Haan, 2010; Rieckmann, 2012; Wiek, Withycombe y Redman, 2011; Novo y Murga-Menoyo, 2015) Asimismo, la EDS exige métodos participativos que motiven a los estudiantes y les doten de la autonomía y el impulso necesarios para cambiar su conducta y promover la adquisición de capacidades, actitudes, valores, habilidades y competencias fundamentales, orientadas hacia un futuro sostenible.

Desde el punto de vista metodológico, la EDS se basa en métodos de enseñanza-aprendizaje innovadores que permiten un aprendizaje auto-organizado y que promueven el desarrollo de la iniciativa y responsabilidad propia del estudiante (Hauenschild y Bolscho, 2005). De igual forma, la ESDS aspira a la utilización de una variedad de métodos y favorece una forma de enseñanza que promueva un aprendizaje auto-dirigido, basado en proyectos, interdisciplinario, colaborativo y con una relevancia real y práctica (De Haan, 2004; Michelsen y Fischer, 2015). Más 
LORENA I. LITZNER ORDÓÑEZ Y WERNER RIEß

LA EDUCACIÓN PARA EL DESARROLLO SOSTENIBLE EN LA UNIVERSIDAD BOLIVIANA. PERCEPCIONES DEL PROFESORADO

aún, según Hauenschild y Bolscho (2005) se caracteriza a través de métodos que incluyen formas de instrucción orientadas a proyectos, situaciones concretas y con una orientación práctica y de acción; así como también métodos interdisciplinarios que promocionen las competencias para la sostenibilidad y la participación. La EDS

busca una pedagogía transformadora y orientada a la acción, y se caracteriza por aspectos tales como el aprendizaje autodidacta, la participación y la colaboración, la orientación hacia los problemas, la inter y transdisciplinariedad, y la creación de vínculos entre el aprendizaje formal e informal (UNESCO, 2017, p. 7).

Algunas de las formas innovadoras de aprendizaje y métodos en el marco de la EDS y la ESDS son, entre otros, el juego de roles, talleres y conferencias orientadas al futuro, mesas redondas, la técnica de worst-case-scenario, simulaciones por computadora, trabajo con modelos, obtención de información con la ayuda de nuevos medios (por ejemplo: uso de bases de datos), técnica de lluvia de ideas y mind-mapping, procedimientos y técnicas de mediación, técnicas de taller como open space (lugar abierto) y demás métodos de planificación y evaluación (Rieß, 2010).

En definitiva, la EDS, en el marco de la educación superior, busca habilitar y transformar a los estudiantes en ciudadanos planetarios (Murga-Menoyo y Novo, 2017) activos y participativos quienes además de contribuir a un futuro sostenible, busquen alcanzar un mundo más equitativo, en paz, tolerante e inclusivo. Esta transformación social, exige una educación de calidad (en todos los ámbitos y niveles) mediante la cual se adquieran conocimientos (saber y entender lo que pasa), y, más allá, se habilite a los estudiantes a sentirse como parte de los procesos de desarrollo y les dote de las herramientas, competencias y valores necesarios para participar activamente de estos procesos de desarrollo sostenible.

\section{LA INVESTIGACIÓN EMPíRICA EN LA EDUCACIÓN SUPERIOR PARA EL DESARROLLO SOSTENIBLE (ESDS)}

El desarrollo sostenible es, sin duda alguna, uno de los desafíos más grandes de nuestro tiempo y representa un desafío también para la investigación científica (Adomßent et al., 2007). La investigación empírica, dedicada a la EDS, desempeña un papel importante con el fin de alcanzar el objetivo ulterior de la investigación: la obtención de nuevos conocimientos. La investigación sobre EDS persigue, según Barth (2016) dos objetivos principales: el primero es un objetivo teórico que busca complementar y puntualizar el concepto de la EDS (Gräsel et al., 2013) y el segundo es un objetivo de implementación práctica, basado en el desarrollo de evidencia y revisión empírica de medidas pedagógicas concretas. Según Kehren (2016), la investigación empírica relativa a la EDS tiene una orientación de aplicación y puesta en práctica, con el objetivo de medir y operacionalizar la EDS. Más aún, la investigación empírica relativa a la EDS, no se centra en el qué (contenido y teorías) del 
desarrollo sostenible; sino más bien en el cómo (método y didáctica) en el proceso de enseñanza - aprendizaje relativo a la sustentabilidad.

La EDS aún no está acompañada de investigación cuantitativa, que aporte conocimientos empíricamente probados y confiables (Rieß, 2010), más bien existen lagunas en la investigación empírica que afectan también al ámbito de la educación superior. La bibliografía, señala en éste contexto, incluso serios vacíos en la investigación (Adomßent y Henze, 2013). Según Cotton et al. (2007) a pesar de que la EDS se está convirtiendo en un modelo cada vez más importante para el desarrollo de planes de estudio en las universidades, es poca la investigación en relación a los docentes universitarios. Por su parte, Michelsen et al. (2011) afirman que, en el ámbito de la formación de profesores, aún no está donde debería estar y corroboran el marcado déficit en cuanto a estudios empíricos relativos a la EDS en éste ámbito.

Cabe mencionar que, con respecto a las propiedades de los estudios empíricos en el ámbito de la educación superior, existen, según Leal Filho, distintos «niveles» (Levels) tanto en la cantidad como en la calidad de dichos estudios (Leal Filho y Manolas, 2012). La bibliografía señala estudios de casos (Case studies) como también estudios en el ámbito europeo como en los Estados Unidos (Mint y Rieckman, 2017), mas podemos evidenciar la falta de estudios empíricos en la región andina de América Latina en el contexto de la EDS en la educación superior (Geli de Ciurana y Leal Filho, 2006). Más aún, de la revisión bibliográfica sistemática realizada por Mint y Rieckmann (2017) para examinar el estado actual en relación a los enfoques y métodos de enseñanza-aprendizaje orientados a la sostenibilidad en la educación superior, resulta que únicamente 21,3\% de los estudios realizados se refieren a datos empíricos.

En el contexto alemán, por ejemplo, la investigación empírica relativa a la EDS, se practica a niveles relativamente bajos. Entre los estudios empíricos podemos mencionar a la Evaluación titulada «Educación para el Desarrollo Sostenible en la formación de Docentes en las Universidades de Baden-Württemberg» (Siegmund y Jahn, 2014). La atención de dicho Estudio se centró en el estado actual de la implementación de la EDS en la formación de profesores en las universidades pedagógicas. Por un lado, se evaluó la consolidación de la EDS en términos institucionales y estructurales, y por otro lado la difusión de los objetivos, contenidos y métodos, como también las posibilidades y limitaciones con respecto a la EDS en la formación del profesorado. El estudio adecuó sus instrumentos de recogida de datos al Marco Modelo (ver figura 1) según Rieß (2006) y concluyó estableciendo la falta de implementación y anclaje estructural de la EDS, de su concepto, fines y métodos, a nivel de formación de profesores en las universidades participantes.

En el contexto latinoamericano, aunque se han llevado a cabo algunos estudios relevantes para la EDS en el ámbito universitario, por ejemplo Colombia (Orozco Silva, 2001) y México (Velazquez, Munguía y Sánchez, 2005), éstos son más bien la excepción en la investigación educativa empírica sobre la EDS en América Latina (Geli de Ciurana y Leal Filho, 2006). Ejemplos relevantes para mencionar son el proyecto 
LORENA I. LITZNER ORDÓÑEZ Y WERNER RIEß LA EDUCACIÓN PARA EL DESARROLLO SOSTENIBLE EN LA UNIVERSIDAD BOLIVIANA. PERCEPCIONES DEL PROFESORADO

ENSU - «Enseñar y aprender de sostenibilidad» realizado en el Ecuador (Rieckmann et al., 2010), el Proyecto "Experiencia de formación del profesorado basada en el contexto ciudad-escuela «en Colombia (Lopera Pérez, 2017) y el «Estudio comparativo del Principio de Participación de la EDS en el marco de la educación superior entre México y Alemania" (Krah, 2017). Por su parte, los resultados del proyecto "Definición de indicadores para la evaluación de las políticas de sustentabilidad en Universidades Latinoamericanas», desarrollado por la Red de Indicadores de Sustentabilidad en las Universidades - RISU ${ }^{4}$ (Benayas et al., 2014), ponen de manifiesto que las universidades latinoamericanas deben hacer aún grandes esfuerzos para avanzar en la inclusión de la sostenibilidad en sus planes de estudio y en la formación integral de los futuros profesionales (Benayas et al., 2014). Asimismo, respecto a la EDS en el ámbito de la educación universitaria, podemos afirmar que una de las barreras, dentro de la investigación educativa, en especial en los países de América Latina, es la falta de investigación empírica. Existe una brecha grande entre los aún escasos programas de investigación empírica, su difusión en la comunidad científica y la puesta en práctica de los resultados utilizables de dichos estudios, programas o proyectos. Más aún, si bien la demanda de integración de la EDS en el contexto de la educación superior es generalizada, hoy por hoy, no existen estudios empíricos sobre el estado actual de la ESDS en el ámbito latinoamericano. En el caso particular de Bolivia, a la fecha, no se cuenta con estudios empíricos en éste contexto. En éste entendido, cabe preguntarse qué sucede en las universidades bolivianas en términos de EDS e identificar las condiciones, ya sea beneficiosas u obstructivas, para la implementación de sus objetivos, contenidos y métodos.

\section{INTRODUCCIÓN AL SISTEMA DE EDUCACIÓN SUPERIOR BOLIVIANO}

Las universidades en Bolivia son tanto públicas como privadas, y se subdividen en instituciones de educación superior autónomas y no autónomas (Martínez Barrientos et al., 2016). Las primeras, gozan de libertad en sus procesos académicos y de desarrollo de políticas, además administran sus recursos de forma autónoma. Por su parte las universidades no autónomas son instituciones educativas privadas y están bajo supervisión y vigilancia estatal. La duración promedio de los estudios universitarios, para la mayoría de las áreas y disciplinas, es de diez semestres y concluye con el grado de Licenciatura. Desde fines de la década de los noventa, el sistema de educación superior boliviano, tanto en el sector público como en el privado, ofrece cursos de postgrado (Rodríguez Ostria y Weise Vargas, 2006) en dos modalidades: en primer lugar, programas de postgrado que otorgan un título

4. En éste proyecto participaron 11 redes universitarias ambientales y 65 universidades de 10 países latinoamericanos: Argentina, Brasil, Chile, Colombia, Costa Rica, Guatemala, México, Perú, República Dominicana y Venezuela (Benayas et al., 2014). 

PERCEPCIONES DEL PROFESORADO

de maestría y/o doctorado y en segundo lugar programas de diplomado. Éstos últimos, son cursos cortos de educación complementaria y de actualización (de 3 a 9 meses) en distintas áreas de conocimiento y no otorgan un título académico.

En el sistema de educación superior boliviano, el grado académico de doctorado no es requisito para el acceso a las cátedras universitarias y otros puestos de responsabilidad académica ${ }^{5}$. Sin embargo, en los últimos años, han aumentado los esfuerzos para capacitar a los docentes universitarios, y se crearon los primeros programas de maestría y diplomado en Educación Superior (Rodríguez Ostria y Weise Vargas, 2006). Al presente, las universidades, tanto públicas como privadas, exigen éstos postgrados como requisito para el desempeño de la función docente.

Una característica de la docencia universitaria en Bolivia, (Martínez Barrientos et al., 2016), es que los profesores universitarios, en su mayoría, no están involucrados en la investigación; ya que el enfoque de las universidades bolivianas está dirigido a la enseñanza (Rodríguez Ostria y Weise Vargas, 2006). El hecho que la mayoría de los docentes universitarios, especialmente en universidades privadas, no trabaja a dedicación exclusiva impide el desarrollo de la investigación científica. Esto es, sin duda, una de las principales deficiencias y limitaciones en la educación superior boliviana.

\section{ESTADO ACTUAL DE LA EDS EN LA EDUCACIÓN SUPERIOR EN BOLIVIA. ESTUDIO EMPÍRICO}

\subsection{Objetivos}

El estudio sobre el statu quo de la EDS en la educación superior, se centró en los docentes de universidades bolivianas, tanto públicas como privadas, sobre la base de las siguientes preguntas de investigación:

¿Cuál es el estado actual de la EDS en la educación superior en Bolivia? Qué aspectos y/o condiciones caracterizan, promueven o benefician la EDS, o, en su defecto, ¿qué obstáculos o barreras impiden su implementación?

¿Qué métodos y técnicas de enseñanza-aprendizaje utilizan los docentes universitarios, a momento de incluir contenidos relevantes a la EDS en el contexto de la educación superior en Bolivia?

Para poder responder al qué (contenidos temáticos) y cómo (métodos) se imparten los contenidos y objetivos de la EDS a nivel superior, se identificaron cuatro variables con el fin de determinar las características estructurales puntuales de la EDS.

5. Hasta el 2014, únicamente 492 docentes (3,3\% de un total de 26.062 docentes) tenían el título de doctorado (Martínez Barrientos et al., 2016). 
LORENA I. LITZNER ORDÓÑEZ Y WERNER RIEß LA EDUCACIÓN PARA EL DESARROLLO SOSTENIBLE EN LA UNIVERSIDAD BOLIVIANA. PERCEPCIONES DEL PROFESORADO

\subsection{Variables}

\section{a) Aspectos personales}

Ésta variable se centra en el desarrollo de un perfil docente con fin de identificar; por ejemplo, el conocimiento de los conceptos de desarrollo sostenible y de la EDS, así como la importancia y relevancia, en lo personal, de temas relativos a la sostenibilidad y a la problemática ambiental. Estos aspectos responden a subinterrogantes como: ¿qué papel juega la EDS para los docentes en el contexto de la educación superior? y ¿qqué valor tiene para el docente universitario la problemática ambiental y la temática del desarrollo sostenible?

\section{b) Aspectos de contenidos temáticos}

Ésta segunda variable, está relacionada con los contenidos temáticos que imparten las cátedras en las universidades bolivianas; se refiere a interrogantes como: ¿qué contenidos temáticos, con relevancia ambiental directa o indirecta, son incluidos en el proceso de enseñanza-aprendizaje? y iqué contenidos son abordados, de forma transversal y sistémica, desde la perspectiva del desarrollo sostenible y de la EDS?

c) Aspectos metodológicos

Una vez identificados los contenidos temáticos relevantes para la sustentabilidad, se vio la importancia de preguntar sobre el método o los métodos utilizados para impartir dichos contenidos; vale decir, ¿cómo se enseñan los temas relativos a la EDS? y ¿qué métodos de enseñanza-aprendizaje y qué medios se usan para transmitir los contenidos relevantes para la EDS y sus objetivos?

\section{d) Aspectos institucionales y estructurales}

La cuarta y última variable se refiere al diagnóstico de la situación institucional y estructural con relación a la EDS. Por ejemplo: ¿qué obstáculos y barreras juegan un papel al momento de hacer realidad una EDS en el contexto universitario?, ¿qué aspectos institucionales y estructurales se pueden diagnosticar como importantes para la implementación de la EDS? y por último ¿qué apoyo, en términos de conocimiento fundamental (saber cómo), cooperación interna o externa existe o es deseada en las instituciones educativas?

\subsection{Instrumento de recogida de datos}

Los datos de la investigación se recogieron mediante un cuestionario para docentes universitarios. En el proceso de diseño del cuestionario, se planificó una prueba preliminar de pre-pilotaje (Edelmann et al., 2012). Todos los ítems desarrollados fueron testeados por expertos en la materia, en términos de validez del contenido, facilidad de comprensión y grado de dificultad (Bortz, 2009). Sobre esta base, los ítems fueron revisados y optimizados. La traducción de los cuestionarios fue necesaria ya que éstos fueron diseñados en idioma alemán. 
El objetivo del diseño del cuestionario, fue construir un instrumento de recogida de datos que incorpore los elementos estructurales resaltados en la figura 1, para proporcionar una visión general y amplia de la EDS en el contexto de la educación superior boliviana. Para el presente estudio, se adaptaron los elementos estructurales del Modelo Marco según Rieß (2006) a la educación superior tal cuál fue efectuado en el estudio Siegmund y Jahn (2014) sobre el estado de la implementación de la EDS en universidades alemanas. Dicho Modelo Marco (referido a la educación escolar) contiene varios elementos estructurales que están interconectados de forma compleja e interdependiente. Cada elemento estructural se puede examinar individualmente, o se puede estudiar la interacción entre dos o más de sus elementos. El cuestionario fue concebido de tal forma que los bloques de preguntas se refieren a las cuatro variables ya mencionadas, que a su vez están basadas en los elementos estructurales del Modelo Marco. El cuestionario incluye un total de 37 ítems, con 11 preguntas abiertas y 26 preguntas cerradas.

Figura 1. Modelo marco

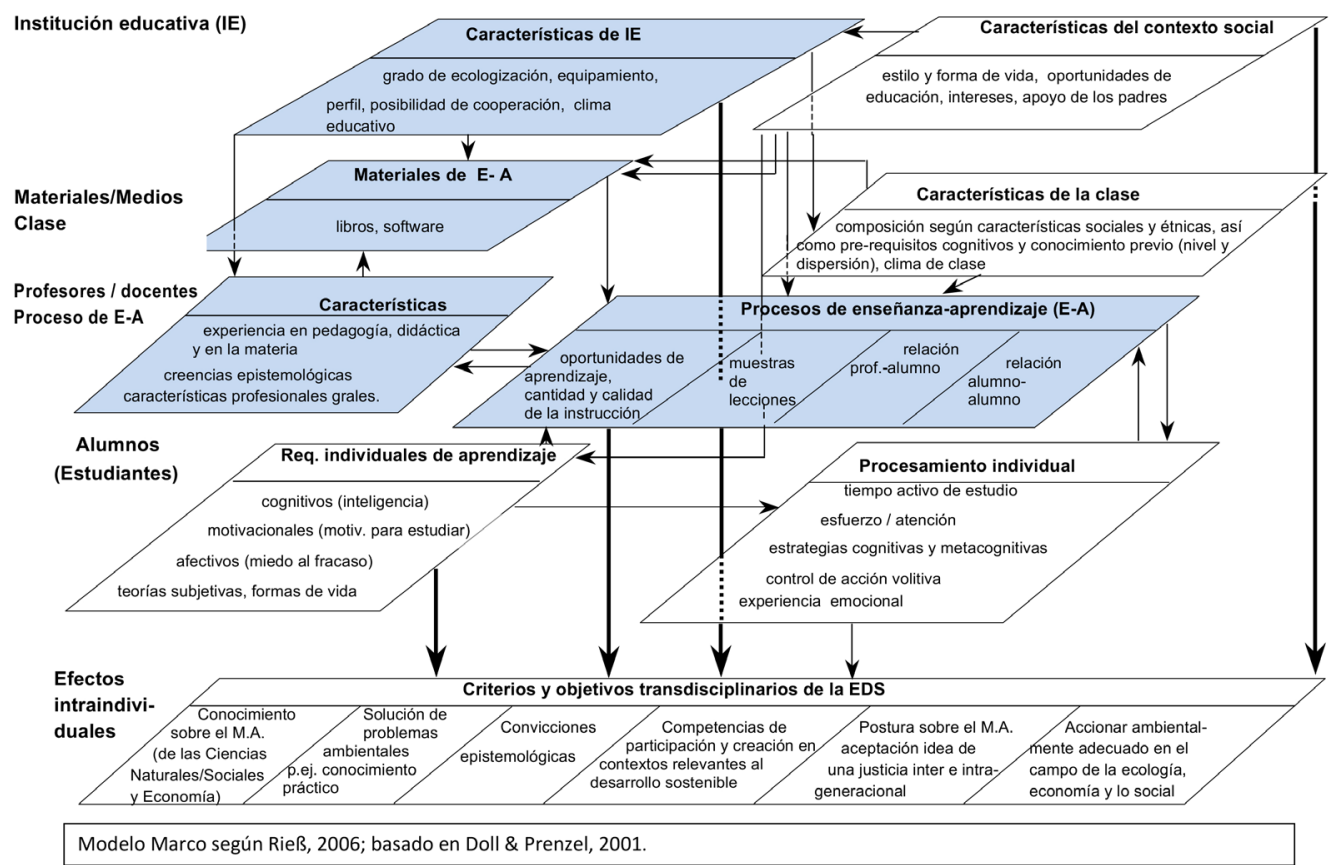


LORENA I. LITZNER ORDÓÑEZ Y WERNER RIEß LA EDUCACIÓN PARA EL DESARROLLO SOSTENIBLE EN LA UNIVERSIDAD BOLIVIANA. PERCEPCIONES DEL PROFESORADO

La tabla 1 presenta la estructura del cuestionario.

Tabla 1. Estructura del cuestionario

\begin{tabular}{|c|c|c|c|}
\hline VARIABLES & $\begin{array}{l}\text { ELEMENTO } \\
\text { ESTRUCTURAL }\end{array}$ & $\begin{array}{c}\text { CUESTIONARIO/ } \\
\text { BLOQUES DE PREGUNTAS }\end{array}$ & ITEMS \\
\hline $\begin{array}{l}\text { 1. Aspectos } \\
\text { personales }\end{array}$ & $\begin{array}{l}\text { Características } \\
\text { de los profesores } \\
\text { / docentes } \\
\text { universitarios }\end{array}$ & $\begin{array}{l}\text { A: Preguntas generales } \\
\text { B: Bloque de preguntas sobre } \\
\quad \text { Desarrollo Sostenible y EDS } \\
\text { E: Postura general sobre Desarrollo } \\
\quad \text { Sostenible y la EDS }\end{array}$ & $\begin{array}{c}1-15 \\
16-19 \\
37 \\
\end{array}$ \\
\hline $\begin{array}{l}\text { 2. Aspectos de } \\
\text { contenidos } \\
\text { temáticos }\end{array}$ & $\begin{array}{l}\text { Proceso de } \\
\text { enseñanza- } \\
\text { aprendizaje }\end{array}$ & $\begin{array}{l}\text { C1: Preguntas sobre la EDS en } \\
\text { el proceso de enseñanza - } \\
\text { aprendizaje }\end{array}$ & $20-24$ \\
\hline $\begin{array}{l}\text { 3. Aspectos } \\
\text { metodoló- } \\
\text { gicos }\end{array}$ & $\begin{array}{l}\text { Proceso de } \\
\text { enseñanza- } \\
\text { aprendizaje } \\
\text { Materiales de } \\
\text { enseñanza - } \\
\text { aprendizaje }\end{array}$ & $\begin{array}{l}\text { C2: Métodos de enseñanza - } \\
\text { aprendizaje }\end{array}$ & $25-27$ \\
\hline $\begin{array}{l}\text { 4. Aspectos } \\
\text { instituciona- } \\
\text { les y estruc- } \\
\text { turales }\end{array}$ & $\begin{array}{l}\text { Características de la } \\
\text { institución educativa }\end{array}$ & $\begin{array}{l}\text { D: Condiciones marco para la EDS / } \\
\text { barreras y obstáculos }\end{array}$ & $28-36$ \\
\hline
\end{tabular}

\subsection{Descripción de la muestra}

Debido a las características de la investigación (recursos, movilidad, tiempo), se optó por una muestra por conveniencia (Bortz, 2009). La elección de las universidades participantes se limitó a dos ciudades. Para la selección de instituciones de educación superior, se utilizaron las listas oficiales de universidades (MinEDU, 2016) del Sistema de Educación Superior boliviano. La participación de las universidades fue voluntaria, más se consideró la importancia de incluir universidades tanto estatales como privadas, para poder hacer afirmaciones válidas y garantizar la representatividad; es decir, para asegurar la confiabilidad y validez de los resultados de la encuesta (Bortz, 2009).

De un total de 850 cuestionarios, 403 (47,4\%) fueron devueltos con datos válidos. El 62,8\% de los participantes son hombres y $37,2 \%$ mujeres. La edad promedio es de 43,9 y oscila entre 21 y 79 años de edad. Los encuestados tienen en promedio 14,4 semestres de experiencia laboral $(\mathrm{SD}=13,1)$. En cuanto a la relación laboral, los resultados obtenidos muestran una característica del sistema universitario boliviano; a saber, 70,2\% de los participantes ejerce sus funciones de forma complementaria a otra actividad laboral principal, mientras que 29,8\% trabaja a dedicación exclusiva. 

PERCEPCIONES DEL PROFESORADO

La tabla 2 muestra las características de la muestra con relación al número (una o más) y al tipo (estatal o privada) de universidades a las que pertenecen los docentes encuestados:

Tabla 2. Comparación: número y tipo de universidad

\begin{tabular}{|c|c|c|c|c|}
\hline \multirow{2}{*}{} & \multicolumn{4}{|c|}{ NÚMERO DE UNIVERSIDADES } \\
\cline { 2 - 5 } & \multicolumn{2}{|c|}{$\begin{array}{c}\text { UNA UNIVERSIDAD } \\
(\mathrm{n}=305)\end{array}$} & $\begin{array}{c}\text { DOS O MÁS UNIVERSIDADES } \\
(\mathrm{n}=98)\end{array}$ \\
\cline { 2 - 5 } & PRIVADA & ESTATAL & $\begin{array}{c}\text { PRIVADA / } \\
\text { PRIVADA }\end{array}$ & $\begin{array}{c}\text { PRIVADA / } \\
\text { ESTATAL }\end{array}$ \\
\hline Número/ & $248 /$ & $57 /$ & $57 /$ & $41 /$ \\
Porcentaje & $81,3 \%$ & $18,7 \%$ & $58,2 \%$ & $41,8 \%$ \\
\hline Total Porcentaje & $61,6 \%$ & $14,1 \%$ & $14,1 \%$ & $10,2 \%$ \\
\hline Total & \multicolumn{3}{|c|}{$75,7 \%$} & \multicolumn{2}{c|}{$24,3 \%$} \\
\hline
\end{tabular}

En cuanto al tipo de universidad, se observa que $24,3 \%$ de los docentes encuestados ejerce sus funciones en dos o más universidades; 14,1\% trabaja en dos o más universidades privadas y 10,2\% trabaja en los dos tipos de universidades simultáneamente. Trabajar en dos universidades estatales al mismo tiempo no es posible por razones geográficas ${ }^{6}$. Por su parte, la mayoría de los encuestados, vale decir $75,7 \%$, trabaja en una universidad, ya sea ésta privada $(61,6 \%)$ o pública $(14,1 \%)$.

\subsection{Resultados}

A continuación se presentan los resultados destacables, de acuerdo a las cuatro variables objeto de investigación:

\section{a) Aspectos personales}

En lo que respecta al concepto de la EDS, 42,7\% de los encuestados afirma conocer dicho concepto, mientras $57,3 \%$ no lo conoce. A los docentes que contestaron de forma afirmativa, se les solicitó nombrar uno o dos fines u objetivos de la EDS. En promedio, fueron mencionados 1,7 objetivos por persona. Los objetivos mencionados fueron categorizados en concordancia con los objetivos de la EDS mencionados en la bibliografía especializada (De Haan y Harenberg, 1999; Rieß, 2010; Michelsen y Fischer, 2015) con una escala de 3 valores que van desde: (0) no es objetivo de la EDS, (1) objetivo mediato de la EDS y (2) objetivo inmediato de la EDS. Los datos fueron doblemente codificados para calcular la concordancia de codificación, obteniendo un valor de.86 (Cohens-Kappa/ =«casi perfecto«) (Landis

6. Bolivia cuenta con 10 universidades estatales, una por Departamento. Únicamente la cuidad de La Paz cuenta con dos universidades públicas (MinEDU, 2016). 

PERCEPCIONES DEL PROFESORADO

y Koch, 1977). Los resultados muestran que del grupo de profesores universitarios que afirma conocer el concepto de EDS, el 75,3\% efectivamente nombró objetivos de la EDS, de éstos son 38,7\% objetivos mediatos y 36,6\% objetivos inmediatos de la EDS. El 24,7\% restante nombró objetivos que no corresponden a los objetivos de la EDS. La tabla 3 muestra los resultados.

Tabla 3. Categorización de los objetivos

\begin{tabular}{|c|c|c|c|}
\hline \multirow{2}{*}{ OBJETIVO(S) } & \multicolumn{2}{|c|}{ OBJETIVO(S) DE LA EDS } & \multirow{2}{*}{$\begin{array}{l}\text { \% DE LOS } \\
\text { CASOS }\end{array}$} \\
\hline & $\mathrm{N}$ & $\%$ & \\
\hline $\begin{array}{l}\text { 0: El objetivo mencionado no corresponde a } \\
\text { los objetivos de la EDS }\end{array}$ & 58 & $24,7 \%$ & $42,6 \%$ \\
\hline $\begin{array}{l}\text { 1: El objetivo mencionado es un objetivo } \\
\text { mediato de la EDS }\end{array}$ & 91 & $38,7 \%$ & $66,9 \%$ \\
\hline $\begin{array}{l}\text { 2: El objetivo mencionado es un objetivo } \\
\text { inmediato de la EDS }\end{array}$ & 86 & $36,6 \%$ & $63,2 \%$ \\
\hline Total & 235 & $100,0 \%$ & $172,8 \%$ \\
\hline
\end{tabular}

Para obtener datos sobre la percepción del profesorado con relación a EDS se elaboró un ítem de respuesta cerrada ${ }^{7}$. Éste ítem fue contestado únicamente por aquellos docentes que afirmaron incluir temas de EDS en sus cátedras, vale decir $53,1 \%$ del total de la muestra. Los resultados muestran que de éstos profesores, la mayoría (85,4\%, M=1,70) afirman que es posible incluir la EDS en todas las materias. Más aún, 93,6\% (M=1,40) coinciden en que incluir temas de la EDS en la educación universitaria, influye de forma positiva en el comportamiento ambiental de los estudiantes. Asimismo, con una aprobación del 65,0\%, queda claro que la EDS es reconocida, desde el punto de vista de los encuestados, como una tarea de la Universidad. De éstos resultados es interesante evidenciar una inclinación positiva hacia la temática ambiental y de sustentabilidad por parte de aquellos docentes que efectivamente incluyen temas relativos a la EDS. Este hecho refleja la necesidad de impulsar y consolidar ésta predisposición positiva del profesorado para la promoción de los objetivos, contenidos y métodos de la EDS.

En cuanto a la variable personal, se evidencia un claro déficit como consecuencia de la falta de conocimiento sobre: (1) la EDS (42,7\% desconocen el concepto), (2) la Década de la EDS (67,4\% la desconocen) y (3) los materiales de enseñanzaaprendizaje relacionados con la EDS (sólo 9,7\% afirma conocer éstos materiales). Éste déficit de información puede ser contrarrestado a través de cursos y/o seminarios

7. Ítem de respuesta cerrada con una escala de: (1) siempre de acuerdo, (2) de acuerdo la mayor parte de las veces, (3) de acuerdo algunas veces y (4) no de acuerdo. 
de capacitación interdisciplinaria dirigida a los docentes universitarios. Los diplomados en Educación Superior, con su sistema modular, configuran la plataforma ideal para éste tipo de programas de capacitación. Un aspecto importante, que debe ser tematizado en los cursos y programas de capacitación, es el potencial de integración (Rieß y Mischo, 2008, 82) del concepto y objetivos de la EDS en el proceso de enseñanza-aprendizaje en la educación superior.

\section{b) Aspectos de contenidos temáticos}

En éste bloque de preguntas se presentó una lista de temas significativos de la EDS, con el fin de que los encuestados identifiquen aquellos incluidos en sus cátedras, conferencias y seminarios. Se articularon en cuatro bloques temáticos: (1) temas con relevancia ecológica, (2) temas con relevancia económica y/o técnica, (3) temas con relevancia sociocultural y (4) temas con relevancia multidimensional. Los resultados muestran que el 57,8\%, de 370 encuestados, afirma incluir tema(s) pertenecientes a los bloques temáticos mencionados. Tomando en cuenta el total de la muestra (N=403), el 53,1\% de los encuestados afirma incluir los temas listados en sus cátedras universitarias. Si recordamos que únicamente $42,7 \%$ de los docentes afirmó conocer el concepto de la EDS, se cristaliza una discrepancia entre el conocimiento del concepto de la EDS y el tratamiento efectivo de temas relativos a la EDS en el proceso de enseñanza-aprendizaje. En otras palabras, los docentes universitarios efectivamente incluyen temas relevantes para la EDS en sus cátedras, mas no lo hacen bajo la categoría o «etiqueta» de la EDS.

En un siguiente ítem, se solicitó a los docentes que nombraran él o los temas de la EDS tratados en aula. Un total de 220 docentes (54,6\% del total de encuestados) listaron un total de 642 temas. En promedio, un docente, mencionó 2,9 temas. En términos del total de la muestra, fueron mencionados 1,6 temas por docente. Los temas se codificaron ${ }^{8}$ como se indica en la tabla 4.

Tabla 4. Temas y su relevancia con la EDS

\begin{tabular}{|l|c|c|c|}
\hline \multirow{2}{*}{ TEMA(S) } & \multicolumn{2}{c|}{ NÚMERO DE CASOS } & \% EN LOS \\
\cline { 2 - 4 } & $\mathrm{N}$ & $\%$ & CASOS \\
\hline 0: sin relevancia con la EDS & 26 & $4,0 \%$ & $11,6 \%$ \\
\hline 1: con enfoque multidimensional & 176 & $27,4 \%$ & $78,1 \%$ \\
\hline 2: con enfoque predominantemente sociocultural & 171 & $26,6 \%$ & $76,0 \%$ \\
\hline $\begin{array}{l}\text { 3: con enfoque predominantemente económico y/o } \\
\text { técnico }\end{array}$ & 157 & $24,5 \%$ & $69,8 \%$ \\
\hline
\end{tabular}

8. Para calcular el índice de concordancia, se codificaron todos los datos dos veces, obteniendo un valor=.84 "casi perfecto» Cohens-Kappa (Landis y Koch, 1977). 
LORENA I. LITZNER ORDÓÑEZ Y WERNER RIEß LA EDUCACIÓN PARA EL DESARROLLO SOSTENIBLE EN LA UNIVERSIDAD BOLIVIANA. PERCEPCIONES DEL PROFESORADO

\begin{tabular}{|l|c|c|c|}
\hline \multirow{2}{*}{\multicolumn{1}{|c|}{ TEMA(S) }} & \multicolumn{2}{c|}{ NÚMERO DE CASOS } & \multirow{2}{*}{$\begin{array}{c}\text { EN LOS } \\
\text { CASOS }\end{array}$} \\
\cline { 2 - 4 } 4: con enfoque predominantemente ecológico & $\mathrm{N}$ & $\%$ & $49,8 \%$ \\
\hline Total & 112 & $17,4 \%$ & 42 \\
\hline
\end{tabular}

De la tabla anterior se desprende una distribución uniforme de los temas mencionados. Si bien no se puede identificar un dominio definido de alguno de los temas, los resultados muestran que los temas con un enfoque multidimensional predominan levemente con $27,4 \%$. A éstos temas, los siguen temas con un enfoque sociocultural (26,6\%) y aquellos con un enfoque predominantemente económico-técnico (24,7\%). Los problemas con un enfoque ecológico están algo menos representados con un 17,4\%. Únicamente el 4,0\% de los temas mencionados no tienen relación con el desarrollo sostenible o la EDS.

Más allá de la descripción de los resultados, de forma explorativa, se hizo una comparación entre los docentes que incluyen temas de EDS y aquellos que no lo hacen. De la comparación de éstos dos grupos de docentes se evidencia, que aquellos docentes que afirmaron conocer el concepto de desarrollo sostenible (chi-cuadrado $=11,521$, df $=1, \mathrm{p}<.001, \mathrm{n}=368$ ) y el concepto de EDS (chicuadrado $=42,824$, df $=3, \mathrm{p}<.001, \mathrm{~N}=396$ ), incluyen con mayor frecuencia, temas relevantes para la EDS en sus cátedras. Éstos resultados, que son estadísticamente significativos, reflejan claramente la importancia y la necesidad de introducir y tratar los conceptos, contenidos, objetivos y desafíos del desarrollo sostenible y de la EDS (Künzli David, 2007) tanto en los planes de estudios de las universidades, como también en los cursos de capacitación dirigidos a los docentes universitarios.

En cuanto a los resultados relativos a los contenidos temáticos relativos a la EDS, es importante resaltar el hecho de que los temas multidimensionales relacionados con la EDS dominan, aunque ligeramente $(27,4 \%)$, con respecto a los temas con carácter unidimensional. Si bien, éstos resultados se refieren únicamente a la muestra, pueden ser tomados como indicios positivos en el manejo de temas relativos a la EDS. En éste entendido, cabe recomendar la necesidad de promover y reforzar el principio interdisciplinario de la EDS, que implica trabajar de forma multidimensional, desde dos o más perspectivas y disciplinas. En la práctica universitaria, es posible integrar y promover éste principio a través de proyectos interdisciplinarios (de cooperación interna o externa). La administración universitaria juega un papel central en este sentido.

Con el objetivo de analizar si los grupos de docentes que incluyen temas de la EDS y aquellos que no lo hacen, difieren significativamente en cuanto a sus conocimientos, percepción y predisposición hacia la EDS, se llevó a cabo la comparación de los promedios de dos ítems del cuestionario (14 y 37) con un formato de respuesta 

PERCEPCIONES DEL PROFESORADO

con una escala de 1 (siempre de acuerdo) a 4 (no de acuerdo) ${ }^{9}$. Para completar la verificación de la significación estadística y determinar si las diferencias también son prácticamente significativas, se utilizó como indicador la medida de tamaño del efecto $d$ (.2 pequeño,.5 medio y.8 alto) (Cohen, 1988). En tres de las características examinadas, las diferencias entre los grupos fueron significativas (Test-t, $\mathrm{p}<.05$ ).

Los resultados indican que los docentes que incluyen temas relacionados con la EDS en sus cátedras universitarias,

- afirman que la EDS tiene una alta prioridad en su vida laboral como docente,

- están mayormente de acuerdo en que es posible incluir temas relacionados con la EDS en todas las materias, y

- tienen una mayor predisposición para incluir temas relevantes para la EDS, aún tomando en cuenta que esto implica un mayor esfuerzo.

Figura 2. Perfil promedio de docentes con diferentes prácticas de EDS

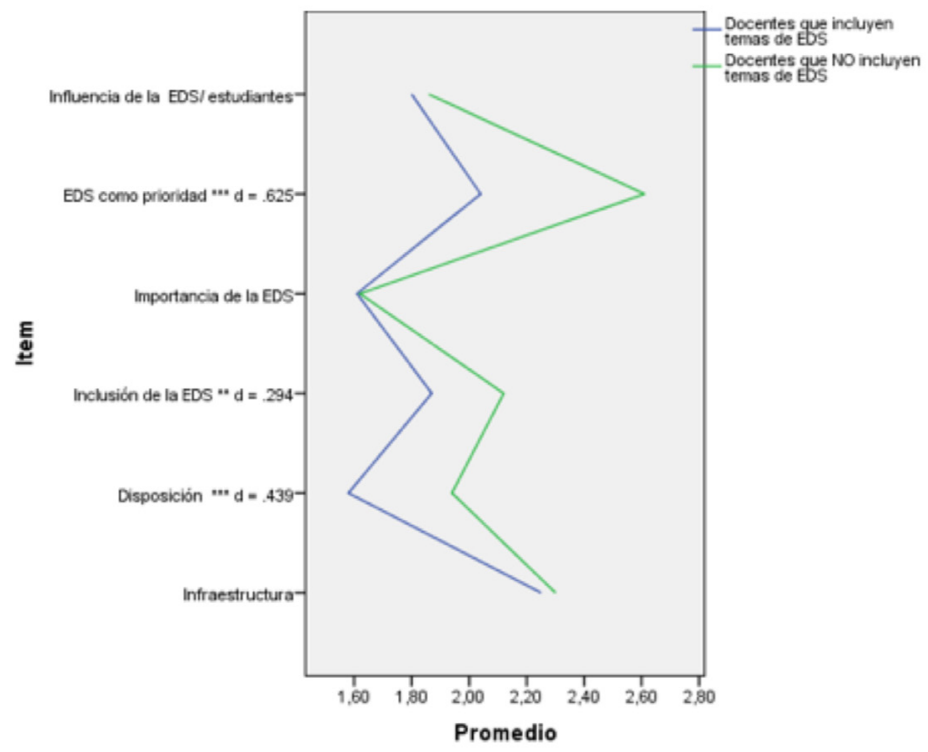

Explicación de la figura: \# $\mathrm{p}<.10,{ }^{*} \mathrm{p}<.05$, ** $\mathrm{p}<.01$, *** $\mathrm{p}<.001$

9. Ítems figura 2: (1) Influencia de la EDS/ estudiantes: Mediante la Educación para el Desarrollo Sostenible se puede influir en el comportamiento ambiental de los estudiantes. (2) EDS como prioridad: La EDS tiene una alta prioridad en mi vida laboral como docente. (3) Importancia de la EDS: Es importante difundir/transmitir los contenidos, métodos, medios y objetivos de la EDS (4) Inclusión de la EDS: Temas sobre Desarrollo Sostenible y EDS deben ser incluidos en todas las materias. (5) Disposición: Estoy dispuesto a incluir temas relativos a la EDS aún cuando represente un mayor esfuerzo. (6) Infraestructura: La universidad tiene una buena infraestructura, biblioteca y medios. 
Los demás resultados, si bien no son estadísticamente significativos, pueden ser considerados como indicios que reflejan una actitud positiva hacia la EDS. Es así que los profesores que enseñan temas relacionados con la EDS:

- señalan que la EDS tiene un impacto positivo en la conciencia ambiental y el comportamiento de los estudiantes relacionados con la sostenibilidad.

- afirman que es importante transmitir el contenido, los métodos, los medios y los objetivos de la EDS a los estudiantes, y por último

- informan sobre una mejor infraestructura universitaria.

c) Aspectos metodológicos

El ítem relativo a los aspectos metodológicos fue contestado por 234 docentes (58,1\% del total de la muestra), quienes mencionaron un total de 1.313 métodos de enseñanza-aprendizaje. Se presentó a los docentes una lista de métodos y técnicas, con la posibilidad de selección múltiple. En promedio, cada uno de los participantes mencionó 5,6 métodos.

El método más mencionado fue el debate. El 69,2\% de los encuestados indicó usar éste método específico para tratar temas de relevancia ambiental. En segundo lugar, se encuentra el trabajo con textos, cuadros, tablas, diagramas, imágenes, pictogramas. Éste último es utilizado por el 60,7\% de los docentes universitarios. 53,4\% de los encuestados utilizan los siguientes dos métodos: (1) trabajo en grupos pequeños y (2) presentaciones usando medios visuales y multimediales. El 39,7\% de los docentes mencionan la técnica del brainstorming y el mapeo mental (mindmapping) como métodos en la enseñanza. En quinto lugar, el 38,5\% de los docentes utilizan el trabajo con música y/o películas. Un porcentaje casi idéntico $(38,0 \%)$ hace uso del trabajo cooperativo en aula para abordar cuestiones relacionadas con la EDS. El 35,9\% de los profesores mencionaron a las presentaciones breves de los estudiantes como método utilizado. La tabla 5 contiene los resultados.

Tabla 5. Métodos de enseñanza-aprendizaje

\begin{tabular}{|l|c|c|}
\hline \multicolumn{1}{|c|}{ MÉTODO / TÉCNICA } & N & $\begin{array}{c}\text { \% DE LOS } \\
\text { CASOS }\end{array}$ \\
\hline Discusiones en aula y/o debates & 162 & $69,2 \%$ \\
\hline Trabajo con textos, tablas, diagramas, pictogramas, gráficos & 142 & $60,7 \%$ \\
\hline Trabajo en grupos pequeños & 125 & $53,4 \%$ \\
\hline Presentaciones con medios audiovisuales & 125 & $53,4 \%$ \\
\hline Lluvia de ideas / mind mapping & 93 & $39,7 \%$ \\
\hline Utilización de música y películas & 90 & $38,5 \%$ \\
\hline Trabajo cooperativo en aula & 89 & $38,0 \%$ \\
\hline Presentaciones cortas de los estudiantes & 84 & $35,9 \%$ \\
\hline Trabajo con periódicos, revistas & 62 & $26,5 \%$ \\
\hline Entrevistas a expertos & 39 & $16,7 \%$ \\
\hline
\end{tabular}


LORENA I. LITZNER ORDÓÑEZ Y WERNER RIEß

LA EDUCACIÓN PARA EL DESARROLLO SOSTENIBLE EN LA UNIVERSIDAD BOLIVIANA. PERCEPCIONES DEL PROFESORADO

\begin{tabular}{|l|c|c|}
\hline \multicolumn{1}{|c|}{ MÉTODO / TÉCNICA } & N & $\begin{array}{c}\% \text { DE LOS } \\
\text { CASOS }\end{array}$ \\
\hline Elaboración de posters, papelógrafos, trabajos artísticos, etc. & 37 & $15,8 \%$ \\
\hline $\begin{array}{l}\text { Observaciones, mediciones e investigación en la naturaleza y al } \\
\text { aire libre }\end{array}$ & 29 & $12,4 \%$ \\
\hline $\begin{array}{l}\text { Visita a otros lugares de enseñanza (p. ej. museo, juzgado, entre } \\
\text { otros) }\end{array}$ & 28 & $12,0 \%$ \\
\hline Juego de roles (p. ej. prácticas forenses). & 26 & $11,1 \%$ \\
\hline Trabajo de protección al medio ambiente & 23 & $9,8 \%$ \\
\hline Trabajo con software especializado & 21 & $9,0 \%$ \\
\hline Excursiones & 19 & $8,1 \%$ \\
\hline Trabajo experimental (energía solar, mediciones, etc.) & 19 & $8,1 \%$ \\
\hline Workshops & 19 & $8,1 \%$ \\
\hline Juegos y otros métodos kinestésicos & 16 & $6,8 \%$ \\
\hline Otros & 16 & $6,8 \%$ \\
\hline Utilización de la técnica de worst-case-escenario & 15 & $6,4 \%$ \\
\hline Elaboración de diarios y/o portafolios de estudio & 14 & $6,0 \%$ \\
\hline Trabajo auto iniciado de los estudiantes & 12 & $5,1 \%$ \\
\hline Proyectos multidisciplinarios con otras facultades & 8 & $3,4 \%$ \\
\hline Total & 1313 & $561,1 \%$ \\
\hline
\end{tabular}

Con respecto a los métodos y procedimientos recomendados por la EDS, los resultados muestran que: $12,4 \%$ de los encuestados utiliza observaciones, mediciones, investigaciones en la naturaleza; 9,8\% opta por trabajos ambientales; 8,1\% realiza trabajos experimentales por ejemplo con energía solar, mediciones de energía, entre otros; 8,1\% de los docentes organiza excursiones con estudiantes y en último lugar $3,4 \%$ realiza y/o participa de proyectos interdisciplinarios (por ejemplo con otras facultades).

Interpretando los resultados sobre los métodos y técnicas utilizados para impartir temas de relevancia ambiental, se evidencia la utilización de una variedad de métodos (en promedio 5,6 métodos), procedimientos y técnicas. No existe dominio de uno o dos métodos y/o técnicas. En cuanto a los métodos específicos de la EDS, éstos son aún utilizados de forma menos frecuente. En éste sentido, vemos la importancia, de dotar a los docentes universitarios con las herramientas metodológicas y didácticas necesarias, que les permitan un acercamiento a los nuevos enfoques didácticos y metodológicos en la práctica docente.

\section{d) Aspectos institucionales y estructurales}

Un aspecto importante del estudio fue identificar las posibles barreras y obstáculos (institucionales, curriculares y/o personales) para la implementación de la EDS en la educación superior. Para éste fin se creó un ítem en formato de respuesta abierta. Las respuestas se categorizaron según recoge la tabla 6. El índice de concordancia 
LORENA I. LITZNER ORDÓÑEZ Y WERNER RIEß LA EDUCACIÓN PARA EL DESARROLLO SOSTENIBLE EN LA UNIVERSIDAD BOLIVIANA. PERCEPCIONES DEL PROFESORADO

de codificación se basó en una doble codificación de los datos con un valor "casi perfecto» =.82 Cohens-Kappa (Landis y Koch, 1977).

Los resultados se pueden resumir de la siguiente manera:

Tabla 6. Categorización de las barreras de la EDS

\begin{tabular}{|c|c|c|c|c|}
\hline CATEGORÍA & RESPUESTA & $\mathrm{N}$ & $\begin{array}{l}\text { \% TOTAL } \\
(\mathrm{N}=403)\end{array}$ & $\begin{array}{c}\% \\
\text { RESPUESTAS } \\
(\mathrm{n}=322)\end{array}$ \\
\hline $\begin{array}{l}\text { Aspectos } \\
\text { institucionales y } \\
\text { estructurales }\end{array}$ & $\begin{array}{l}\text { - Factor tiempo / temas } \\
\text { demandan mayor tiempo } \\
\text { - Cátedras y seminarios } \\
\text { sobresaturados } \\
\text { - Falta de material(es) de } \\
\text { enseñanza y/o bibliografía } \\
\text { especializada }\end{array}$ & 92 & $22,8 \%$ & $28,6 \%$ \\
\hline $\begin{array}{l}\text { Aspectos de } \\
\text { contenidos } \\
\text { curriculares }\end{array}$ & $\begin{array}{l}\text {-Los temas de la EDS no son } \\
\text { contenido de la materia }\end{array}$ & 85 & $21,1 \%$ & $26,4 \%$ \\
\hline "Nada" & -Explícitamente nombrado & 60 & $14,9 \%$ & $18,6 \%$ \\
\hline $\begin{array}{l}\text { Aspectos personales } \\
\text { (docentes) }\end{array}$ & $\begin{array}{l}\text { - Requiere mayor dedicación/ } \\
\text { esfuerzo } \\
\text { - Poca relevancia en lo personal }\end{array}$ & 46 & $11.4 \%$ & $14,3 \%$ \\
\hline $\begin{array}{l}\text { Falta de } \\
\text { información / } \\
\text { conocimiento sobre } \\
\text { la EDS }\end{array}$ & $\begin{array}{l}\text { - Falta de información } \\
\text { - Falta de conocimiento } \\
\text { sobre contenidos y temas de } \\
\text { desarrollo sostenible }\end{array}$ & 24 & $6,0 \%$ & $7,5 \%$ \\
\hline $\begin{array}{l}\text { Aspectos personales } \\
\text { (estudiantes) }\end{array}$ & $\begin{array}{l}\text { - Falta de interés por los temas } \\
\text { de desarrollo sostenible } \\
\text { - Bajo nivel de rendimiento }\end{array}$ & 15 & $3,7 \%$ & $4,7 \%$ \\
\hline Total & & 322 & $79,9 \%$ & $100,0 \%$ \\
\hline
\end{tabular}

- 28,6\% de los encuestados identificaron barreras institucionales y estructurales, como el factor tiempo, las cátedras y seminarios sobresaturados y la falta de material de enseñanza-aprendizaje y/o bibliografía relevante a la EDS.

- 26,4\% de los encuestados señalaron que los contenidos de relevancia para la EDS no son parte del contenido curricular (plan de estudios) o que no tienen relación con el contenido temático del campo o disciplina que enseñan.

- 18,6\% señala explícitamente "nada» como impedimento para tratar los temas de EDS.

- 14,3\%, mencionaron aspectos específicos del docente. Entre éstos, mencionaron que (1) estos temas implican mayor dedicación en tiempo y en esfuerzo y (2) que éstos temas tienen un escaso significado en lo personal. 

PERCEPCIONES DEL PROFESORADO

- 7,5\% de los encuestados señala específicamente no tener los conocimientos suficientes sobre desarrollo sostenible y la EDS.

- 4,7\% de los docentes señala como obstáculo para el tratamiento de éstos temas, que los estudiantes (1) no muestran interés en los temas relacionados con el desarrollo sostenible y que (2) su bajo nivel de rendimiento impide la inclusión de dichos temas.

En cuanto a los aspectos estructurales e institucionales, las universidades deben percibir las barreras mencionadas en el presente estudio, como reales y serias. Queda claro, que las barreras institucionales $(28,6 \%)$ y curriculares $(26,4 \%)$ constituyen obstáculos estructurales que impiden la promoción de la EDS. En éste entendido, vemos la importancia de «llegar» a los docentes que mencionaron que los temas de EDS «no son contenido en mi materia» $(26,4 \%)$ y a aquellos que contestaron explícitamente "nada" (18,6\%). Más aún, es importante que todos los docentes interioricen el principio y potencial de integración, así com la transversalidad e interdisciplinariedad de los temas de sostenibilidad en el proceso educativo.

En cuanto a las condiciones institucionales y estructurales, los resultados confirman una de las características del sistema educativo boliviano; a saber, el predominio de docentes que ejercen sus funciones como actividad complementaria a otra actividad laboral principal (únicamente 29,8\% de los docentes trabaja a dedicación exclusiva). Es así que es recomendable la institucionalización de la carrera docente, tomando en cuenta las siguientes consideraciones: (1) los docentes a dedicación exclusiva, se confrontan no sólo con contenidos, sino también con los métodos didácticos de la educación superior; (2) de ésta forma se crean y fortalecen las estructuras de investigación, innovación y transferencia de conocimientos; (3) los docentes a dedicación exclusiva, por lo general, trabajan en una universidad y, por lo tanto, están familiarizados con las instalaciones, los materiales, la biblioteca, el equipamiento, etc. (4) éstos docentes utilizan, o pueden hacer un mejor uso, de las posibilidades de cooperación de las universidades.

\section{CONSIDERACIONES FINALES}

En el contexto de la educación superior en Bolivia, se reconoce la importancia de implementar el concepto de sostenibilidad y de la EDS en la educación superior, más no es posible hablar de una consolidación estructural del concepto, contenidos, objetivos y métodos de la EDS en éste ámbito. Las insuficiencias institucionales, tales como la falta de infraestructura adecuada, el acceso deficiente o escaso a medios y bibliografía especializada, las limitaciones financieras (recursos/tiempo), y las estructuras de cooperación casi inexistentes, constituyen barreras estructurales en el sistema educativo universitario boliviano. Estamos de acuerdo en que el desafío del desarrollo sostenible en la educación universitaria, no obliga únicamente a reorientar los currículos universitarios, sino que también llama a transformar el modelo 
LORENA I. LITZNER ORDÓÑEZ Y WERNER RIEß LA EDUCACIÓN PARA EL DESARROLLO SOSTENIBLE EN LA UNIVERSIDAD BOLIVIANA. PERCEPCIONES DEL PROFESORADO

educativo, e incluir e impulsar al docente universitario en su función de agente promotor de un proceso educativo activo, participativo, solidario y transformador.

\section{REFERENCIAS BIBLIOGRÁFICAS}

Acosta, A. (2015). Buen Vivir. Vom Recht auf ein gutes Leben. München: Ed. Oekom.

Acosta, A. (2011). Buen Vivir auf dem Weg in die Post-Entwicklung. Ein globales Konzept?. En W. Rätz, T. Von egan-Krieger, B. Muraca, A. Passadakis, M. Schmelzer, y A. Vetter (Eds.). Ausgewachsen! Ökologische Gerechtigkeit, soziale Rechte, gutes Leben -ein Projekt von Attac. (pp. 173-183). Hamburg: VSA-Verlag.

Adomßent, M., Michelsen, G., Rieckmann, M. y Stoltenberg, U. (2007). Die «Sustainable University» als informeller Lernkontext. Zeitschrift für internationale Bildungsforschung und Entwicklungspädagogik, 30 (4), 9-12.

Adomßent, M. y Henze, C. (2013). Hochschulbildung für nachhaltige Entwicklung. En N. Pütz, N. Logemann y M.K.W. Schweer (Eds.), Bildung für nachbaltige Entwicklung. Aktuelle theoretische Konzepte und Beispiele praktischer Umsetzung (pp. 159-181). Frankfurt: Peter Lang Internationaler Verlag der Wissenschaften.

Barth, M. (2016). Forschung in der Bildung für nachhaltige Entwicklung: Entstehung und Verortung eines Forschungszweiges. En M. Barth y M. Rieckmann (Eds.), Empirische Forschung zur Bildung für nachbaltige Entwicklung - Themen, Methoden und Trends (pp. 37-50). Opladen: Verlag Barbara Budrich.

Benayas Del Álamo, J., Alba, D. y Justel, A. (2014). Definición de indicadores para la evaluación de las políticas de sustentabilidad en Universidades Latinoamericanas. Proyecto RISU. Recuperado de: http://www.pnuma.org/educamb/documentos/GUPES/ Proyecto_risu_Final_2014.pdf (Consultado el 5/12/2018).

BID-Banco Interamericano De Desarrollo (1993). Derecho Ambiental Internacional. Santiago de Chile: Editorial BID.

Boff, L. (2009). ¿Vivir mejor o «el buen vivir»? Recuperado de: https://studylib.es/doc/266445/ el-buen-vivir---leonardo-boff (Consultado el 17/01/2019)

Bortz, J. y Döring, N. (2009). Forschungsmethoden und Evaluation für Human- und Sozialwissenschaftler. Heidelberg: Editorial Springer.

Cohen J. (1988). Statistical Power Analysis for the behavioral Sciences. $9^{\mathrm{a}}$ ed. Nueva York: Academic Press. https://doi.org/10.1002/bs.3830330104

Cotton, D.R.E., Warren, M.F., Maiboroda, O. y Bailey, I. (2007). Sustainable development, higher education and pedagogy: a study of lecturer s beliefs and attitudes. Environmental education Research, 13(5), 579-597. https://doi.org/10.1080/13504620701659061

De Haan, G. y Harenberg, D. (1999). Bildung für eine nachhaltige Entwicklung. Gutachten zum Programm, Bonn: BLK.

De Haan, G. (2004). Transferkonzept für das BLK -Modellprogramm «Bildung für eine nachhaltige Entwicklung» (BLK-Programm «21«): Beschlussvorlage für die Projektgruppe Innovationen der BLK. 

PERCEPCIONES DEL PROFESORADO

De Haan, G. (2010). El desarrollo de las competencias relacionadas a la EDS en los marcos institucionales de apoyo. International Review of Education, 56(2), 315-328. https://doi. org/10.1007/s11159-010-9157-9

Edelmann, D., Schmidt, J. y Tippelt, R. (2012). Einführung in die Bildungsforschung. Grundriss der Pädagogik/ Erziehungswissenschaft. Stuttgart: Verlag W. Kohlhammer.

Geli De Ciurana, A. M. y Leal Fihlo, W. (2006). Education for sustainability in university studies. Experiences from project involving European and Latin American Universities. International Journal of Sustainability in Higher Education, 7(1), 81-93. https://doi. org/10.1108/14676370610639263

González-Gaudiano, E. J. (2006). Environmental Education: a field in tension or in transition? Environmental Education Research, 12, 291-300. https://doi.org/10.1080/13504620600799042

González-Gaudiano, E. J. (2008). Configuración y significado: educación para el desarrollo sustentable. En E. J. González-Gaudiano (Coord.), Educación, medio ambiente y sustentabilidad (pp. 213-227). México: Siglo XXI - UANL.

Gräsel, C. (2009). Umweltbildung. En R. Trippelt y B. Schmidt B. (Eds.), Handbuch Bildungsforschung (pp. 845-859). Wiesbaden:VS-Verlag für Sozialwissenschaften.

Gräsel, C., Bormann, I., Schütte, K., Trempler, K. y Fischbach R. (2013). Outlook on Research in Education for Sustainable Development. Policy Futures in Education, 11(2), 115-127. https://doi.org/10.2304/pfie.2013.11.2.115

Hauenschild, K. y Bolscho, D. (2005). Bildung für Nachhaltige Entwicklung in der Schule. Ein Studienbuch. Frankfurt: Peter Lang Internationaler Verlag der Wissenschaften.

Hauenschild, K. y Rode, H. (2013) Bildung für nachhaltige Entwicklung im schulischen Kontext. En N. Pütz, N. Logemann y M.K.W. Schweer (Eds.), Bildung für nachhaltige Entwicklung. Aktuelle theoretische Konzepte und Beispiele praktischer Umsetzung (pp. 61-82). Frankfurt: Peter Lang Internationaler Verlag der Wissenschaften.

Hauff, V. (1987). Unsere gemeinsame Zukunft. Der Bericht der Weltkommission für Umwelt und Entwicklung (Brundtland Bericht), Greven: Eggenkamp.

Irwin, R. (2008). "Postneoliberalismo»: de la educación ambiental a la educación para la sustentabilidad. En E. J. González-Gaudiano (Coord.), Educación, medio ambiente y sustentabilidad (pp. 176-212). México: Siglo XXI - UANL.

Kehren, Y. (2016). Bildung für nachbaltige Entwicklung - Zur Kritik eines pädagogischen Programms. Baltmannsweiler: Schneider Verlag Hohengehren GmbH.

Krah, J.M. (2017). Partizipative Hochschulbildung für nachbaltige Entwicklung. Implementation und Institutionalisierung in Mexiko und Deutschland. Ludwigsburg: Pädagogische Hochschule Ludwigsburg.

Künzli David, C. (2007). Zukunft mitgestallten. Bildung für nachhaltige Entwicklung Didaktisches Konzept und Umsetzung in der Schule. Bern: Haupt Verlag.

Landis, J. R. y Koch, G. G. (1977). The measurement of observer agreement for categorical data. Biometrics, 33, 159-174. https://doi.org/10.2307/2529310

Leal Filho, W. (2010). Universities and Climate Change. Introducing Climate Change to University Programmes. Heidelberg: Editorial Springer.

Leal Filho, W. y Manolas, E. (2012). Implementing Sustainable Development in Higher Education. En F. Goncalves, R. Pereira, W. Leal Filho y U. Miranda Azeteiro (Eds.), Umweltbil- 
LORENA I. LITZNER ORDÓÑEZ Y WERNER RIEß LA EDUCACIÓN PARA EL DESARROLLO SOSTENIBLE EN LA UNIVERSIDAD BOLIVIANA. PERCEPCIONES DEL PROFESORADO

dung, Umweltkommunikation und Nachhaltigkeit. Contributions to the UN Decade of Education for Sustainable Development (pp. 47-70). Frankfurt: Peter Lang Internationaler Verlag der Wissenschaften.

Lopera Pérez, M. (2017). Experiencia de formación del profesorado basada en el contexto ciudad-escuela. Revista Iberoamericana de Educación / Revista Ibero-americana de Educação, 74(1), 41-58.

Lukman, R. y Glavic, P. (2007). ¿What are the key elements of a sustainable university? Clean Technology and Environmental Policy, 9,103-114. https://doi.org/10.1007/s10098-0060070-7

Martínez Barrientos, A., Santillán Butrón, S. y Loayza Melgarejo M. (2016). Educación Superior en Iberoamérica. Informe Nacional: Bolivia.

Michelsen, G., Adomßent M., Bormann, I., Burandt S. y Fischbach, R. (2011). Indikatoren der Bildung für nach haltige Entwicklung - ein Werkstattbericht. En Deutsche-UNESCO Kommission- (DUK) (Eds.), Bad Homburg: Editorial VAS. Recuperado de: https://www. bne-portal.de/sites/default/files/Indikatoren_2520der_2520BNE.File_0.pdf (Consultado el 15/01/2019).

Michelsen, G. y Fischer, D. (2015). Bildung für nachhaltige Entwicklung. Wiesbaden: HessischeLandeszentralefürPolitischeBildung.

MinEdu-Ministerio de Educación del Estado Plurinacional de Bolivia (2016). Guía de Universidades bolivianas. Recuperado de: http://www.minedu.gob.bo/files/GUIA -UNIVERSIDADES.pdf (Consultado 21/1/2019).

Mora Penagos, W.M. (2009). Educación ambiental y educación para el desarrollo sostenible ante la crisis planetaria: demandas a los procesos formativos del profesorado. Tecné, Episteme y Didaxis, 26, 7-35.

Murga-Menoyo, Mª A. y Novo, M. (2017). Sostenibilidad, desarrollo "glocal» y ciudadanía planetaria. Referentes de una pedagogía para el desarrollo sostenible. Teoría de la Educación. Revista Interuniversitaria, 29(1), pp. 55-78. https://doi.org/10.14201/teoredu2915578

Novo, M. y Murga-Menoyo, $M^{a}$. A. (2015). The Processes of Integrating Sustainability in Higher Education Curricula: A Theoretical-Practical Experience Regarding Key Competences and Their Cross-Curricular Incorporation into Degree Courses. En W. Leal Filho (Eds.), Transformative Approaches to Sustainable Development at Universities (pp. 119-133). Berlin: Editorial Springer.

ONU (1992). Agenda 21. Recuperado de: http://www.un.org/spanish/esa/sustdev/agenda21/ agenda21spchapter36.htm (Consultado el 15/01/2019)

Orozco Silva, L. E. (2001). Educación Superior- desafío global y respuesta nacional. Tomos I y II. Colombia: Universidad de los Andes.

Rieckmann, M., Adomßent, M. y Aguirre, P. (2010). Das internationale Seminar «Bildung für eine nachhaltige Entwicklung und Biodiversität» in Ecuador - ein Ort Globalen Lernens? Zeitschrift für internationale Bildungsforschung und Entwicklungspädagogik, 33, 21-26.

RIeckmann, M. (2012). Educación superior orientada hacia el futuro: ¿Qué competencias clave se deberían fomentar mediante la enseñanza y la educación universitaria? Futures, 44(2), 127-135. 

PERCEPCIONES DEL PROFESORADO

Rieß, W. y Apel, H. (eds.) (2006). Bildung für eine nachbaltige Entwicklung. Aktuelle Forschungsfelder und-ansätze. Wiesbaden: VS-Verlag für Sozialwissenschaften. https://doi. org/10.1007/978-3-531-90192-3

Rieß, W. (2010). Bildung für nach haltige Entwicklung. Theoretische Analysen und empirische Studien. Münster: Waxmann Verlag GmbH.

Rodríguez Ostria, G. y Weise Vargas, C. (2006). Educación Superior Universitaria en Bolivia -Estudio Nacional. Cochabamba: Iesalc-Unesco-Editorial Talleres Gráficos «Kipus».

Sauvé, L. (2006). La educación ambiental y la globalización: desafíos curriculares y pedagógicos. Revista Iberoamericana de Educación, 41, 83-101.

Siegmund, A. y Jahn, M. (2014). Abschlussbericht zur Evaluation «BNE in der Lehramtsausbildung an badenwürttembergischen Hochschulen» im Rahmen des Projekts «Lernen über den Tag hinaus - Bildung für eine zukunftsfähige Welt «im Auftrag des Ministeriums für Kultus, Jugend und Sport Baden-Württemberg. Heidelberg.

UNESCO (2017). Educación para los objetivos del desarrollo sostenible. Objetivos de aprendizaje. Recuperado de: http://unesdoc.unesco.org/images/0025/002524/252423s.pdf (Consultado el 25/11/2017).

Velázquez, L., Mungía, N. y Sánchez, M. (2005). Deterring sustainability in higher education institutions: An appraisal of the factors which influence sustainability in higher education institutions». International Journal of Sustainability in Higher Education, 6, 383-391. https://doi.org/10.1108/14676370510623865

Wiek, A., Withycombe, L. y Redman, C. L. (2011). Key competencies in sustainability: a reference framework for academic program development. Sustainability Science, 6(2), 203-218. https://doi.org/10.1007/s11625-011-0132-6 